\title{
Effectiveness of the Use of the Adaptogen Trekrezan in the Cultivation of African Catfish
}

\author{
Elena Spirina, Elena Romanova*, Ludmila Shadyeva, Vasily Romanov \\ Ulyanovsk State Agrarian University named after P.A. Stolypin, Ulyanovsk, 432017, Russia
}

\begin{abstract}
Growing fish in closed water supply installations with a high planting density leads to accumulation of waste products, turning the habitat into toxic. Growing fish in such an environment leads to stress and increases the stress on their liver, which provides the body with detoxification of metabolites. To reduce stress, adaptogens are used, which increase endurance and survival, increase adaptive plasticity, and strengthen the immunity of fish. We used an adaptogen called Trekrezan. The work aim was to study the morphofunctional changes in liver and its microarchitectonics in African catfish grown using Trekrezan. The analysis of morphofunctional changes in liver allows us to judge the general physiological state of fish body. The liver index of African catfish grown without the use of Trekrezan is significantly higher in both males and females, compared to African catfish grown with Trekrezan, which indicates an intensification of metabolism. Analysis of histological structure of African catfish liver (Clarias gariepinus), grown without the use of the Ttrekrezan, revealed abnormalities of the parenchymal layer of liver with signs of fatty dystrophy, signs of necrotic changes in liver cells, hemorrhages in vascular region, a violation of polarity in the structure of hepatocytes. In species raised in an environment with Trekrezan, these abnormalities are absent, since Trekrezan, due to the activation of cellular and humoral immunity, leads to decrease in intoxication, provides strengthening of immunity, and increases the body's resistance to unfavorable factors of medium.
\end{abstract}

\section{Introduction}

Growing fish in an artificial environment is accompanied by "extreme" living conditions for them, as their waste products are toxic and provide additional stress on the body $[1,2]$. So, in the artificial environment there are food leftovers and products of fish metabolism, decomposition of which leads to an increase in concentration of nitrogen and phosphorus. Nitrogen is present in the form of ammonia, which is oxidized by oxygen to form nitrate- ions, which provide formation of methemoglobin anemia, in which nitrates interact with blood hemoglobin to carry out a redoxreaction. In this case, deoxyhemoglobin is oxidized to methemoglobin, and nitrite ions are reduced to nitrogen monoxide by taking electrons. When interacting with reduced hemoglobins, nitric monoxide forms stable complexes. Methemoglobin anemia, which occurs due to the influence of nitrates on fish body, can change metabolism, suppressing antioxidant defense systems with subsequent abnormalities of cell membranes and lead to a decrease in cellular immunity.

Due to the effect of toxicants on the fish body, there are changes in morphophysiological characteristics of fish, since ensuring survival in the environment occurs at the expense of additional energy costs for decontamination of toxic substances in the environment [3], primarily in liver. Due to the fact that liver in fish body provides detoxification of toxic substances formed in body or coming from outside, the study of morphofunctional state of liver and the analysis of microarchitectonics of liver cells of fish grown in artificial environment is relevant.

To evaluate the degree of favorable external conditions on fish body, method of morphophysiological indicators proposed by S. S. Schwartz is used [3-5]. Changes in fish habitat factors have a direct or indirect effect on the energy balance of body and lead to changes in morphofunctional parameters of fish body, including liver. Therefore, the analysis of morphofunctional changes in liver of fish should be used to assess the factors of their habitat.

Recently, African catfish has become one of the most promising objects grown in artificial conditions [1, 2], since the peculiarities of its biology allow it to be grown in RAS, pools at a water temperature of $25-30{ }^{\circ} \mathrm{C}$ and a fairly low oxygen content in water, due to the fact that it has a well-developed epibranchial organ that allows it to breathe atmospheric oxygen.

Growing African catfish (Clarias gariepinus) in an artificial environment requires intensification of production, which is often accompanied by stress due to compaction of planting and other factors, so it is necessary to reduce stressful conditions and increase the adaptability of fish body $[1,6]$. Therefore, to increase the intensity of basin aquaculture, feed with adaptogens is

\footnotetext{
* Corresponding author: vvr-emr@yandex.ru
} 
used [7-9], due to the fact that the degree of assimilation of such feed increases and the immunity of fish increases. The use of adaptogens contributes to the mobilization of fish body, as they affect cellular and tissue metabolism. Adaptogens have an immunomodulatory effect due to the synthesis of immunoglobulins and thymus-dependent cells [10-12], as well as an anabolic effect. The main advantage of using adaptogens is their harmlessness to the body and their wide stimulating physiological effect [13-15]. An effective adaptogen is Trekrezan [16], the effect of which is similar to ginseng, Eleutherococcus, golden root, etc. [17-19].

Trekrezan is effective in protecting against pain, temperature and nervous stimuli, increases resistance to stress, accelerates regeneration of liver tissue, striated heart tissue and skeletal muscle [16-17], strengthens the immune system and provides protection of internal organs from toxicants, infectious agents due to cellular and humoral immunity, interferon production. Trekrezan increases antioxidant activity and is able to eliminate immune deficits that occur in the case of inflammatory diseases. Trekrezan is characterized by energystimulating effect [18-20].

The aim of the work was to study the effect of Trecrezan on morphofunctional parameters of African catfish liver when grown in an artificial environment.

\section{Materials and methods}

The object of the study was African catfish at 6 months of age (Figure 1), which were kept in pools with a volume of $3.5 \mathrm{~m} 3$. The cultivation of African catfish was carried out on the basis of the Department of Biology, Veterinary Genetics, Parasitology, Ecology, Aquatic Bioresources and Aquaculture of Ulyanovsk SAU.

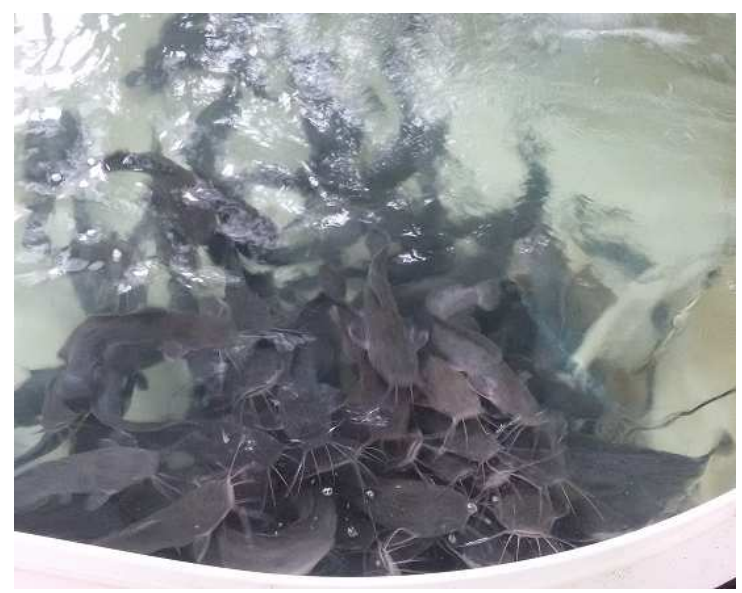

Fig. 1. Growing Clarias gariepinus in UISAU

The water temperature in pools was maintained at $25-26{ }^{\circ} \mathrm{C}$, and the oxygen content was $4 \mathrm{mg} / \mathrm{l}$. For feeding, we used compound feed of the brand "Aqarex SOM", depending on the growth of African catfish, fractions with a diameter of 4, 5, $6 \mathrm{~mm}$ were used.

2 groups of individuals were formed: control and experimental. Individuals of the experimental group were given food sprayed with a solution of Trekrezan at a dosage of $0.03 \mathrm{~g} / \mathrm{kg}$. Individuals of the control group were given food without the addition of Trekrezan.

The liver index was determined to assess the physiological state of fish [3-5]. For this purpose, the total body weight of fish was determined by weighing it on an electronic scale, after opening, fish liver was weighed with an accuracy of $0.001 \mathrm{~g}$. The determination of the African catfish liver index was made by dividing liver weight by body weight and multiplying by 1000 .

To analyze the microarchitecture of liver, histoprobes were selected and further immersed in a solution of $10 \%$ formalin. The production of histopreparations was carried out according to traditional methods [20]. Histopreparations were stained using Mayer's hematoxylin and eosin. The drug was coated with a Sub$\mathrm{X}$ Mounting medium. The histopreparations were examined using an Axio Imager.M2 microscope, analyzed and processed using ZEN pro. The material for histoprerates was collected from three individuals from the control and 6 experimental groups.

\section{Results}

At the first stage of the study, we determined the liver indicator in male and female African catfish bred with Trecrezan and the control group bred without it. The following results were obtained (Figure 2, 3).

When analyzing the data obtained, significant differences are noticeable in African catfish males (Figure 2), since the liver index of control group males is higher, compared to the liver index of males grown using Trekrezan.

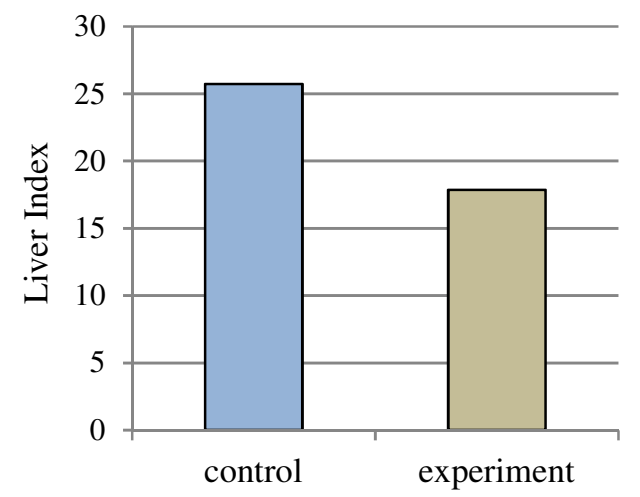

Fig. 2. African catfish liver Index (males)

Similar changes are observed in females. The liver index of females raised without Trekrezan was significantly higher compared to females who were fed with Trekrezan (Figure 3). Liver in fish body provides detoxification of toxic substances coming from external environment by decontaminating blood.

In endoplasmic reticulum of hepatocytes, toxic compounds are neutralized, deactivated, and eliminated from body by increasing molecules polarity and reducing their solubility in lipid-like substances. Bilipid layer of endoplasmic reticulum membrane of hepatocytes contains built-in enzymes that provide microsomal oxidation due to which external substances coming from 
environment or forming in fish body are oxidized and transferred from fat-soluble to water-soluble state with subsequent removal from the body.

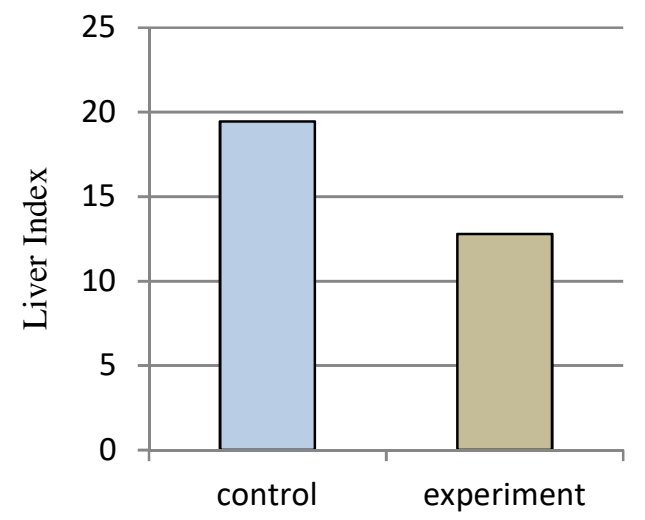

Fig. 3. African catfish liver Index (females)

The use of Trekrezan as a food additive for African catfish grown in an artificial environment is promising, due to the fact that Trekrezan reduces the degree of detoxification leads to energy savings in the fish body and as a result contributes to the intensification of fish growth. While the African catfish of the control group, grown without the use of Trekrezan, intensify their metabolism, they have a higher liver index, to reduce the degree of negative effects on the body.

At the next stage of the study, a histological analysis of the African catfish liver was performed in the experimental and control groups. The fish of the experimental group received the adaptogen Trekrezan during feeding, the fish of the control group were raised without Trekrezan.

Histological analysis of the African catfish liver of the experimental group showed the presence of hepatocyte trabeculae forming a tubular structure (Figure 4) with a central lumen of sinusoid capillaries (Figure 5). The parenchymal layer of liver includes several layers of connective tissue, with virtually no lobes in structure (Figure 4).

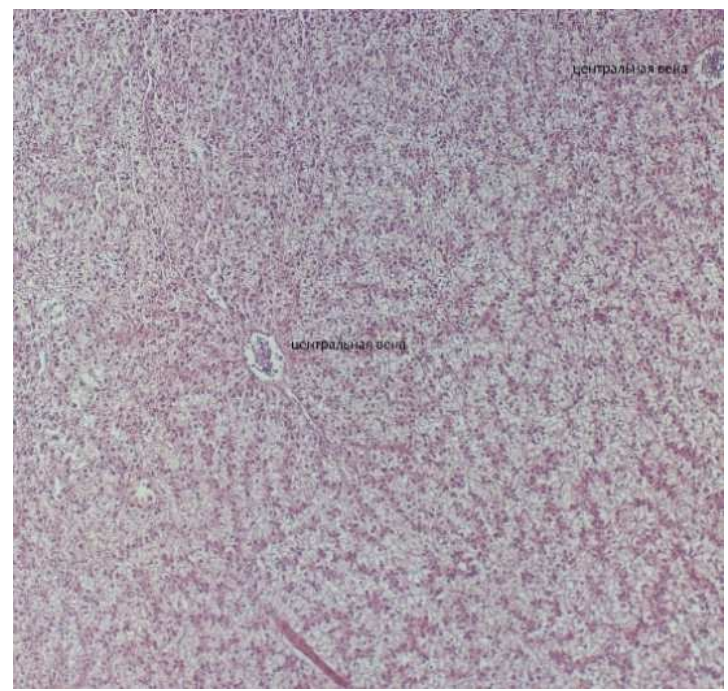

Fig. 4. Features of liver structure of African catfish grown with Trekrezan (10x10).
In addition, there are no typical portal tracts. When considering the histopreparation of the African catfish liver of experimental group, grown on feed with the addition of Trekrezan, groups of several bile ducts are found. Histopreparations of liver of catfish of experimental group contain an arteriole and a central vein. Examination of liver cells of experimental group shows a polarity, due to the fact that the nucleus in liver cells of fish grown using the adaptogen Trekrezan is shifted to the basal part of the cell, while glycogen is visible on the apical part of the cell (Figure 5). Histopreparations of the liver of fish grown using Trekrezan include parenchymal cells, characteristic Ito cells and macrophages are visible.

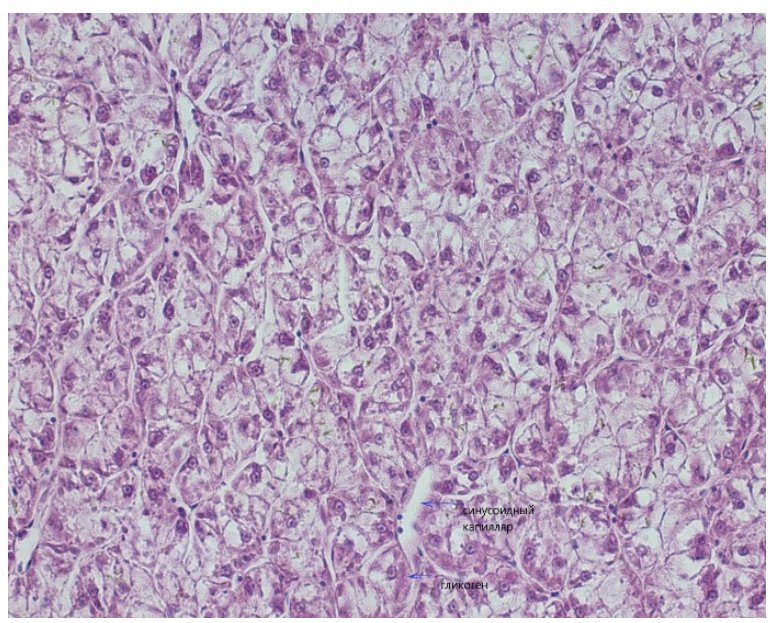

Fig. 5. African catfish liver histopreparation in the experimental group $(10 \times 40)$

Analysis of African catfish histopreparations of the control group grown without adaptogen Trekrezan revealed abnormalities of parenchymal layer of fish liver (Figure 6), indicating obvious dystrophic changes (Figure 6,7). On the African catfish histopreparations of the control group, signs of necrotic changes in hepatocytes are noticeable, traces of hemorrhages in the vessels of parenchymal layer are observed (Figure 6,7).

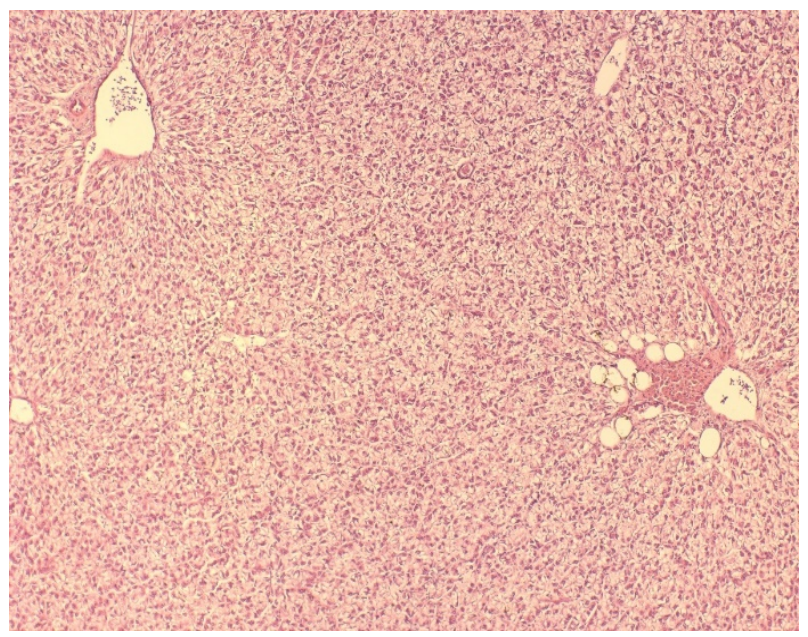

Fig. 6. Histopreparation of African catfish of the control group, with an increase in 10x10. Hepatocytes have fat vacuolation, 
necrotic changes in parenchyma with traces of hemorrhage in the vascular area

When considering the structural features of hepatocytes, there is a violation of the polarity, when the nucleus is localized in the cell center, there are separate red blood cells in the vessels (Figure 7).

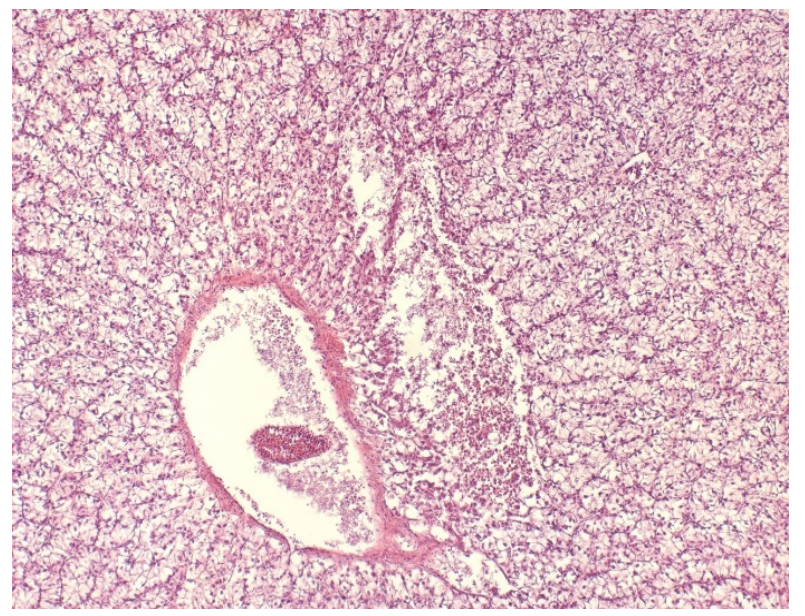

Fig. 7. Histopreparation of African catfish that did not receive Trekrezan. Increase 10x20. Major necrotic disorders in the vascular group.

\section{Discussion}

The analysis of morphofunctional changes in liver and its histopreparations in African catfish grown in an artificial environment indicates the prospects of using the adaptogen Trekrezan as a biologically active substance of feed, since Trekrezan helps to reduce the liver index and the fullness of the liver vessels, reduces dystrophic changes in liver cells, and increases the adaptation of fish in an artificial environment. Trekrezan increases the immunity of African catfish by activating cellular and humoral immunity, reduces the impact of toxicants formed in the body or coming from the external environment when grown in an artificial environment, transforms metabolic products, reducing their toxicity, thereby contributes to the protection of hepatocytes in toxic exposure.

The body liver of fish carries out the process of detoxification, transforming the products of metabolism, while it is important to form non-toxic compounds that would not cause toxic damage to hepatocytes, easily destroyed and excreted from the body.

Therefore, the use of Trekrezan as a dietary supplement helps reduce the morphofunctional and structural disorders of African catfish liver when grown in an artificial environment, as it reduces the impact of toxic metabolites that lead to a violation of microarchitectonics of liver cells.

\section{Conclusions}

Analyzing the morphofunctional and histological changes in the structure of the African catfish liver, we came to the conclusion that it is advisable to use Trekrezan as a dietary supplement, since Trekrezan has a positive effect on the African catfish liver, reduces morphofunctional and toxic changes in liver of catfish raised in an artificial environment. The liver of African catfish grown without the use of the Trekrezan adaptogen has histological disorders of the liver parenchymal layer with signs of fatty dystrophy and necrotic changes in hepatocytes, traces of hemorrhages in the liver vessels are visible. Studies of the histological features of the African catfish liver of the control group indicate necrotic changes and loss of polarity of liver cells; red blood cells were present in the liver vessels, which indicates morphofunctional disorders of liver of African catfish grown in an artificial environment.

Therefore, the use of the adaptogen Trekrezan for the improvement and immunomodulation of fish body when grown in an artificial environment is promising.

\section{Acknowledgments}

The Russian Foundation for Basic Research has supported our study with the grant No. 18-416-730005.

\section{References}

1. E.M. Romanova, V.N. Lyubomirova, V.V. Romanov, M.E. Mukhitova, T.M. Shlenkina, Egypt. J. of aquatic res. 44(4), 315-319 (2018)

2. E. Spirina, E. Romanova, V. Romanov, V. Lyubomirova, L. Shadyeva, T. Shlenkina, L. Rakova, IOP Conf. ser. Earth and Envir. Sci., 403, 012220 (2019)

3. S.S. Shvarts, V.S. Smirnov, L.N. Dobrinsky, Tr. Ierizh, 387 (1968)

4. A. Renold, G. Crepaldi, G. Possa, Acad. Press, 6(2), 1-6 (1981)

5. S.K. Arndt, J. Fish. Biol., 57, 3 (2000)

6. P.D. Shabanov, I.V. Zarubina, A.Yu. Ryleev et al., 5th Int. Congr. of Pathophysiol., Vol. 171 (2006)

7. A.V. Bolekhan, A.Yu. Ryleev, I.V. Zarubina et al., 4th Int. Conf., Vol. 94 (2006)

8. R.B. Weisberg, J. Clin. Psychiat., 70(2), 4-9 (2009)

9. M. Bentzon-Tilia, E.C. Sonnenschein, L. Gram, Microb. Biotechnol., 9(5), 576-584 (2016)

10. H. Ghomrassi, O.B. Braiek, K. Hani, T. Ghrairi, Y. Choiset, T. Haertlé, J.-M. Chobert, Diseases of aquatic organ, 118(1), 31-43 (2016)

11. X. Xiong, G. Huang, Y. Peng, X. Liu, J. of fish. of China, 40(1), 73-82 (2016)

12. M.E. Yonar, S.M. Yonar, M.Z. Çoban, M. Eroğlu, Envir. Toxicol., 29(2), 155-164 (2014)

13. I.I. Rudneva, Compar. Biochem. and physiol., Part C: pharmacol., toxicol. and endocrinol., 118(2), 255-260 (1997)

14. E. Calcagno, P. Durando, M.E. Valdés, L. Franchioni, M.D.L.T. Bistoni, Physiol. \& Behav., 158, 68-75 (2016) 
15. E. Kulczykowska, H. Kalamarz-Kubiak, M. Gozdowska, E. Sokołowska, Compar. Biochem. and Physiol., Part A: Molec. \& Integr. Physiol., 218, 1-7 (2018)

16. M. Fenech, Mutat. Res., 455, 81-95 (2000)

17. J. Mersh, M.N. Beauvais, P. Nagel, Mutat. Res., 371, 47-55 (1996)
18. Y. Kaminsky, E. Kosenko, G. Aliev, Cardiovasc. and hematol. agents in med. chem., 14(1), 49-52 (2016)

19. M.E. Yonar, Ecotoxicol. and Envir. Safety, 151, 4954 (2018)

20. D.E. Korzhevsky, Fund. of Histol. Techn., 95 (2010) 